Le prix du lait de brebis chez nous aujourd'hui ne dépasse guère en moyenne 0 fr. 80 le litre et le pays en possède une production annuelle de 2.500 .000 hectolitres en moyenne, dont presque la moitié pourrait être utilisée en lait caillé pour l'exportation. De la sorte, on posséderait à l'étranger du véritable kvasseno mleko bulgare avec toutes ses qualités diététiques et thérapeutiques, soit pour les hôpitaux, soit pour l'usage personnel.

\title{
REVUE
}

\section{UN PROCÉDÉ AUTOMATIQUE POUR LAFABRICATION DE LA POUDRE DE LAIT}

\author{
par G. GÉNIN \\ Ingénieur chimiste E. P. C.
}

Dans la préparation des produits alimentaires, ainsi que dans les nombreuses fabrications de produits chimiques, le contrôle des opérations les plus délicates peut être aujourd'hui réalisé par des appareils entièrement automatiques dont l'emploi permet d'obtenir des économies substantielles. En effet la présence de ces appareils autorise une réduction du personnel, permet de rendre continues de nombreuses fabrications et évite, par un contrôle meilleur des opérations, des malfaçons et des pertes de matière.

Parmi les produits que les industries alimentaires sont appelées à traiter, il én est peu d'aussi délicat que le lait, et la fabrication de la poudre de lait est une opération qui porte non seulement sur une matière première difficile à traiter, mais qui en outre est une opération délicate à mener en elle-même. Par exemple, les risques de surchauffe peuvent détruire les vitamines essentielles du lait et en même temps entraîner une décomposition de la caséine et favoriser l'apparition d'un goût défectueux du produit.

De nombreuses méthodes de concentration et de déshydratation du lait ont été déjà décrites. Beaucoup d'entre elles permettent d'obtenir des produits satisfaisants. Parmi ces méthodes, le mode opératoire connu sous le nom de procédé Douthitt a été un de ceux employés avec le plus de succès. Il est décrit dans les brevets américains 1.078 .848 de $1913,1.107 .784$ de $1914,1.157 .935$ de 1915 , 1.266 .013 de 1918 et 1.392 .656 de 1921.

Mais ce procédé, comme tout procédé reposant sur l'emploi de la température, nécessite un contrôle très précis, si on veut éviter les risques de surchauffe. Rappelons qu'il consiste essentiellement à concentrer partiellement le lait par un courant d'air chaud, puis à compléter la déshydratation en pulvérisant le lait épaissi dans une 
chambre parcourue par un courant d'air chauffé à une température suffisante. A différents stades du procédé, il faut surveiller avec beaucoup de soin la marche des opérations, pour maintenir la température, ainsi que d'autres variables d'ailleurs, entre des limites très étroites.

Une firme américaine est parvenue à rendre ce procédé automatique par l'emploi d'un grand nombre de dispositifs de contrôle appropriés. Le lait cru est tout d'abord chauffé à $55^{\circ}$ environ dans un réchauffeur Jensen, puis passe dans un séparateur de crème. On le reçoit ensuite dans deux euves dans lesquelles il est maintenu à une température de $80^{\circ}$ environ. A cet effet, il circule continuellement entre un second réchauffeur et les réservoirs, ce qui permet de régler beaucoup plus étroitement sa température.

De ces réservoirs, le lait chaud est dirigé au moyen d'une pompe dans un appareil à concentrer, le niveau du liquide dans ce dernier appareil étant maintenu constant grâce à un système de flotteurs qui ouvrent ou obturent l'orifice d'arrivée du liquide. Le lait aspiré à la partie inférieure de l'appareil à concentrer passe dans un échangeur de température qui porte sa température à exactement $72^{\circ}$; on le renvoie ensuite à l'appareil à concentrer où se trouve un distributeur qui le répartit sur toute la surface d'un atomiseur qui occupe la partie supérieure de l'appareil à concentrer. Le lait tombe goutte à goutte et se concentre, l'air chaud qui arrive dans l'appareil et qui provient d'une autre partie de l'installation éliminant une partie de l'eau qu'il contient.

Sous cette forme partiellement concentrée, le lait formerait très facilement des gâteaux sur les surfaces chauffantes des réchauffeurs si on permettait à la température de s'élever, même très légèrement. Aussi il faut réaliser un contrôle absolu de la température si on veut éviter l'obturation des conduites de l'appareil.

De la partie inférieure de l'appareil à concentrer, le lait est aspiré par une pompe hydraulique et pulvérisé sous une pression d'environ $200 \mathrm{~kg}$. par centimètre carré au moyen d'un appareil à atomiser situé à la partie supérieure d'un eyclone. Dans cet appareil, il rencontre un courant d'air sec et chaud qui a été soigneusement filtré pour éliminer toutes les impuretés et les poussières. La quantité d'air entrant dans l'appareil, ainsi que sa température, doivent être rigoureusement contrôlées; afin que l'eau contenue dans les gouttelettes de lait soit totalement éliminée. La forme en eyclone de cet appareil permet aux particules solides de lait d'être en quelque sorte aspirées vers la partie inférieure du cyclone. L'air qui s'échappe de l'appareil est encore chaud, il contient de petites particules de lait, aussi on le dirige vers l'appareil à concentrer, afin 
qu'on puisse utiliser la chaleur résiduelle qu'il contient et éviter de perdre les petites particules de lait qui ont été entraînées.

Etant donné les dimensions considérables du cyclone, l'installation doit occuper deux étages, mais par contre, la eonduite d'un appareil de ce genre ne nécessite qu'un homme dont le travail consiste simplement à vérifier le bon fonctionnement des appareils de contrôle et à placer le lait en poudre dans des empaquetages appropriés.

C'est surtout au voisinage du réchauffeur destiné à maintenir la température du lait à $72^{\circ}$ que la présence du surveillant est nécessaire et le règlement prévoit qu'il doit toutes les 15 minutes vérifier le bon état de cet appareil. On double d'ailleurs ce contrôle humain d'un contrôle automatique qui règle l'admission de la vapeur dans le réchauffeur en fonction de la température du lait qui circule dans eet appareil. 'La sensibilité de l'appareil de contrôle est telle. qu'il est possible de maintenir la température du lait à moins de un demidegré près.

\section{BIBLIOGRAPHIE ANALYTIQUE}

\section{LES LIVRES}

\section{Wright (Norman C.), - An inquiry into the drinking habits of} children of school age, with special reference to milk drinking (Enquête sur les boissons habituelles des écoliers et en particulier sur le lait). The Hannah Dairy Research Institute, bull. no 7, Kirkhill, Ayr, 1936. 1 br. eartonnée de 50 pages.

Des efforts très divers ont été faits, pendant ces dernières années, pour augmenter la consommation du lait. Des améliorations ont été apportées à la qualité du lait pour augmenter la confiance du consommateur dans sa pureté et son innocuité ; des méthodes de production plus hygiéniques ont été utilisées; on a cherché à diminuer les maladies dans les troupeaux laitiers; on a adopté des méthodes spéciales de traitement comme la pasteurisation. En même temps, dans la plupart des pays, les Pouvoirs publics ont essayé de généraliser la consommation du lait en utilisant la publicité.

La mesure la plus importante prise en Grande-Bretagne à cet égard a été la distribution gratuite, ou à prix réduit, de lait aux enfants fréquentant les écoles subventionnées. Cette mesure, mise en application en automne 1934, a eu aussitôt le plus grand succès. En 1935, plus de 300.000 enfants des écoles subventionnées écossaises (soit près de la moitié de la population seolaire) recevaient du lait, les chiffres correspondant pour l'Angleterre et le Pays de Galles étant de 2.750 .000 enfants.

Malheureusement, aucune enquête préalable au sujet des boissons habi. tuelles des écoliers n'avait été effectuée, de sorte qu'il n'était pas possible de savoir si la mesure prise était couronnée de succès ou non. C'est pourquoi une enquête était nécessaire pour obvier à cette omission. Une enquête analogue 